\title{
Incorporation of $\mathrm{Sb}$ in InAs/GaAs quantum dots
}

\author{
S. I. Molina, a) A. M. Sánchez, A. M. Beltrán, D. L. Sales, and T. Ben \\ Departamento de Ciencia de los Materiales e I.M. y Q.I., Facultad de Ciencias, Universidad de Cádiz, \\ Campus Río San Pedro, s/n, Puerto Real, 11510 Cádiz, Spain
}

M. F. Chisholm, M. Varela, and S. J. Pennycook

Materials Science and Technology Division, Oak Ridge National Laboratory, Oak Ridge, Tennessee 37831, USA

P. L. Galindo

Departamento de Lenguajes y Sistemas Informáticos, CASEM, Universidad de Cádiz, Campus Río San Pedro, s/n, Puerto Real, 11510 Cádiz, Spain

A. J. Papworth ${ }^{\text {b) }}$ and P. J. Goodhew

Department of Engineering, University of Liverpool, Liverpool L69 3GH, United Kingdom

J. M. Ripalda

Instituto de Microelectrónica de Madrid (CNM, CSIC), Isaac Newton 8, 28760 Tres Cantos, Madrid, Spain

(Received 17 October 2007; accepted 29 November 2007; published online 27 December 2007)

\begin{abstract}
The formation of a quaternary InGaAsSb alloy is shown to occur in the core of epitaxial $\mathrm{GaSb}$ capped InAs/GaAs quantum dots emitting at $1.3 \mu \mathrm{m}$. The existence of the four constituent elements is demonstrated by using spatially resolved low-loss electron energy loss spectroscopy and aberration-corrected high angle annular dark field scanning transmission electron microscopy. The intermixing process giving rise to the formation of this quaternary alloy takes place despite the large miscibility gap between InAs and GaSb binary compounds, and is probably driven by the existence of strain in the quantum dots. (C) 2007 American Institute of Physics. [DOI: 10.1063/1.2826546]
\end{abstract}

Telecommunication applications currently use InGaAsP/InP semiconductor diode lasers emitting at 1.3 and $1.55 \mu \mathrm{m}$. Huge research efforts have been dedicated to develop alternative fabrication technologies for growing epitaxial materials on inexpensive large-area GaAs substrates. This advance would enable the extended use of optical fiber communications in local area networks. ${ }^{1}$ The use of selfassembled InAs/GaAs quantum dots (QDs) has been one of the more investigated approaches to solve this technological challenge. ${ }^{2}$ A recent promising approach to fabricate optoelectronic devices emitting at telecom or even longer wavelengths is the growth of $\mathrm{Ga}(\mathrm{As}) \mathrm{Sb}$ on InAs/GaAs quantum dots, with ${ }^{3}$ or without ${ }^{4-7}$ the intermediate growth of a GaAs barrier thin layer separating the InAs and the Ga(As)Sb layers. The introduction of an antimony exposure step during ${ }^{8}$ or after $^{3}$ the QD's growth has also proven to be an effective solution to obtain a redshift in the emission wavelength. More importantly, this approach has achieved an enormous increase in room temperature emission intensity for the redshifted wavelengths. However, there is a lack of knowledge about the structural and compositional changes associated with these phenomena. The difficulty to incorporate antimony inside InAs and InGaAs quantum dots and quantum wells has repeatedly been reported in the literature. ${ }^{9-11}$ In this work, we show clear evidence of $\mathrm{Sb}$ incorporation inside GaSb capped InAs quantum dots with room temperature luminescence at the technologically important wavelength of $1.3 \mu \mathrm{m}$.

\footnotetext{
${ }^{a)}$ On sabbatical leave at Materials Science and Technology Division, Oak Ridge National Laboratory, Oak Ridge, TN 37831, USA. Electronic mail: sergio.molina@uca.es.

${ }^{\mathrm{b}}$ Present address: Gatan, Liverpool, U.K.
}

The sample investigated was grown by molecular beam epitaxy on GaAs (001) substrates. InAs QDs were observed by reflection high energy electron diffraction after deposition of $1.65 \mathrm{ML}$ of InAs at $510{ }^{\circ} \mathrm{C}$ substrate temperature and at a $0.02 \mathrm{ML} / \mathrm{s}$ growth rate. The total InAs deposited was 2.6 ML followed by $2.2 \mathrm{ML}$ of GaSb immediately after InAs QD growth. The sample was then capped with an $80 \mathrm{~nm}$ thick GaAs layer. The temperature was held at $510{ }^{\circ} \mathrm{C}$ until the first $10 \mathrm{~nm}$ of GaAs had been deposited, at which point the temperature was ramped up to $580^{\circ} \mathrm{C}$. The As and $\mathrm{Sb}$ beam equivalent pressures were $1.7 \times 10^{-6}$ and $3.0 \times 10^{-7} \mathrm{mbar}$, respectively. In our molecular beam epitaxy (MBE) system, these values are, respectively, equivalent to $1.8 \times 10^{15}$ and $3.2 \times 10^{14}$ atoms $/\left(\mathrm{s} \mathrm{cm}^{2}\right)$.

The composition of the grown QDs was studied by high resolution scanning transmission electron microscopy (STEM), using a high-angle annular dark field (HAADF) detector and spatially resolved electron energy loss spectroscopy (EELS) in two dedicated scanning transmission electron microscopes. HAADF studies were carried out at $300 \mathrm{kV}$ in a VG HB603U STEM microscope equipped with a Nion aberration corrector. The inner detector angle is $58 \mathrm{mrad}$ and the probe forming aperture semiangle is about $22 \mathrm{mrad}$. EELS analyses were performed at $100 \mathrm{kV}$ using a VG HB601UX STEM equipped with the Gatan ENFINA ${ }^{\mathrm{TM}}$ parallel EELS system. EEL spectra were taken using the low-loss region $(<50 \mathrm{eV})$ with an energy resolution of $0.35 \mathrm{eV}$ (measured in terms of the full width at half maximum of the zero loss peak), using a collection aperture of $1.34 \mathrm{mrad}$ and an electron probe size of $0.8 \mathrm{~nm}$. EEL spectra were also acquired in the core-loss region but the nearness of several In, Ga, Sb, and As edges made it impossible to obtain significant elemental maps. This problem is avoided by analysing the low-loss region of the spectra. The sample was also 


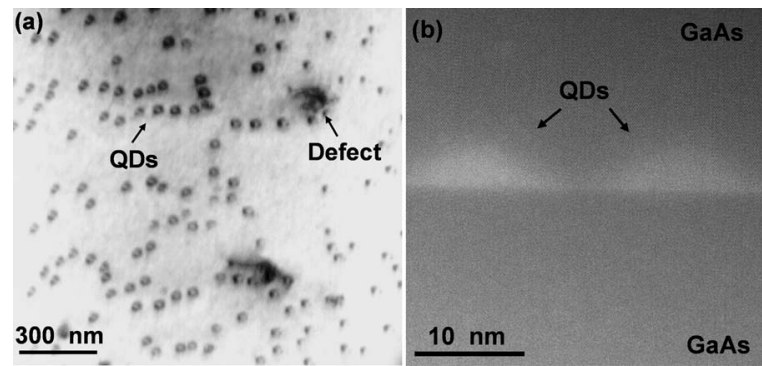

FIG. 1. (a) PVTEM image showing the distribution of QDs. (b) Crosssectional HAADF-STEM images of two QDs.

studied by conventional transmission electron microscopy (TEM) in plan view (PV) and cross sectional orientations to measure the density of the QDs and to analyze the presence of structural defects. Specimens were prepared for TEM and STEM studies by following standard procedures (mechanical thinning and ion milling).

The PVTEM image of Fig. 1(a) shows the typical distribution of QDs in the sample. The density of QDs is 8.5 $\times 10^{9} \mathrm{~cm}^{-2}$. A few structural defects were observed, such as the one marked. Their density amounts to about $10^{7} \mathrm{~cm}^{-2}$. Figure 1(b) shows a low magnification cross-sectional HAADF-STEM image of a couple of QDs. The brighter intensity inside the QDs suggests that they are formed by an alloy with a higher average $Z$ number than GaAs. This alloy therefore has to consist of either InGaAs or InGaAsSb. It is well known that the sides of the QDs lie along $\langle 110\rangle$ directions, thus the base and sloping sides of the QDs are parallel to the electron beam direction. It is immediately clear that the thin layer of $\mathrm{GaSb}$ that was deposited onto the previously formed InGaAs QDs has interdiffused, since no thin bright line is seen covering the sloping sides to the QDs, and there is no evidence for a GaSb thin layer between the islands. The $\mathrm{GaSb}$ coverage in this experiment is below the reported critical thickness for GaSb QD nucleation on $\mathrm{GaAs}(001) .{ }^{12}$ From the HAADF images, we can conclude that the deposited GaSb has interdiffused significantly, but it is unclear if it has been incorporated into the QDs or if it has just been intermixed with the GaAs layer alone.

In order to analyze the existence of intermixing of the GaSb with the InGaAs alloy of the QDs, detailed analytical STEM measurements were performed in the QDs. Figure 2 shows the $\mathrm{Ga}$, In, As, and $\mathrm{Sb}$ elemental maps obtained by low-loss EELS. This analysis has been carried out by using the method explained in Ref. 13. The low-loss spectrum contains features corresponding to the zero loss peak followed by features corresponding to single or multiple scattering events. Major features in the single scattering distribution are the plasmon peak at $16 \mathrm{eV}$, and edges due to excitation of the In $N_{4,5}(18 \mathrm{eV}), \mathrm{Ga} M_{4,5}(20 \mathrm{eV}), \mathrm{Sb} N_{4,5}(31 \mathrm{eV})$, and As $M_{4,5}(40 \mathrm{eV})$ shells. The plasmon peak is removed during the calculation of the imaginary part of the dielectric function $\varepsilon_{2}(E)$ which allows a better background fit. ${ }^{14} \varepsilon_{2}(E)$ contains information about the optical absorption of the material including transition from $d$ levels to the conduction band. By mapping the intensity of $\mathrm{Ga} 3 \mathrm{~d}$ and $\mathrm{As} 3 \mathrm{~d}$, an elemental distribution in the material can be obtained from the background subtracted spectrum images, taking GaAs as a reference for $\mathrm{Ga}$ and As. Figure 2 shows the elemental distribution obtained, following this procedure, for Ga, As, In, and $\mathrm{Sb}$. In and $\mathrm{Sb}$ maps were obtained from $\mathrm{Ga}$ and As maps as $\mathrm{Sb}$. In and Sb maps were obtained from Ga and As maps as detected in the QDs.
Downloaded 30 Mar 2009 to 161.111.235.57. Redistribution subject to AlP license or copyrig

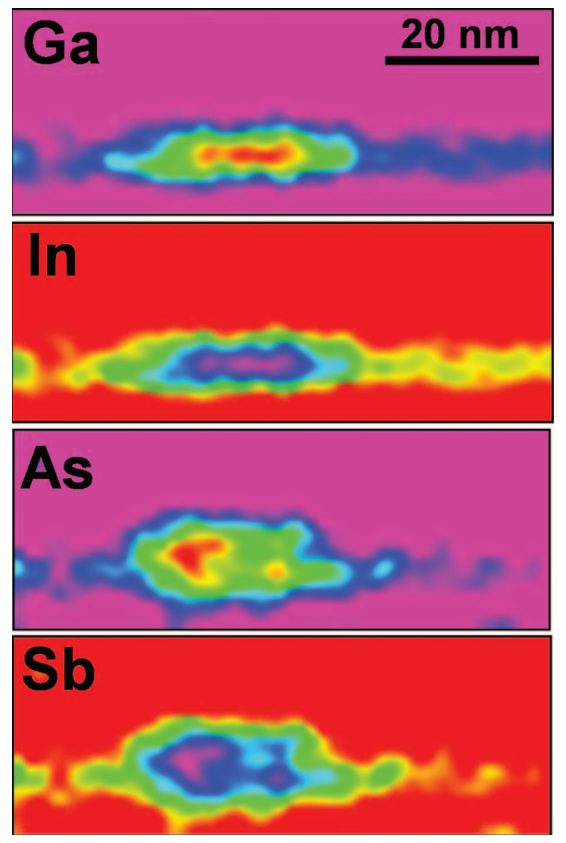

FIG. 2. (Color) Ga, In, As, and Sb elemental maps obtained from the analysis of the low-loss energy region of the spatially resolved background subtracted EEL spectra acquired from the same QD. Rainbow color code is used with red and violet colors representing the minimum and maximum concentrations, respectively. Ga and As maps were determined taking GaAs as a reference for $100 \%$ of both elements. In and $\mathrm{Sb}$ maps were determined as 100 minus the Ga and As maps, respectively. Concentrations of Ga and As in the range $\left[100-2 \sigma_{i}, 100\right]$ are shown in violet, while concentrations of In and $\mathrm{Sb}$ in the range $\left[0,2 \sigma_{i}\right]$ are shown in red. $\sigma_{i}$ is the standard deviation of the concentration for the element $i$ ( $\mathrm{Ga}$ or As) measured from a region consisting of pure GaAs. $\sigma_{\mathrm{In}}$ and $\sigma_{\mathrm{Sb}}$ are assumed to be equal to $\sigma_{\mathrm{Ga}}(4.1 \%)$ and $\sigma_{\mathrm{As}}(5.1 \%)$, respectively. Maximum concentrations of $\mathrm{Sb}$ and $\mathrm{In}$ (violet color) are $41.1 \%$ and $43.9 \%$, respectively.

$100-c_{\mathrm{Ga}}$ and $100-c_{\mathrm{As}}$ where $c_{\mathrm{Ga}}$ and $c_{\mathrm{As}}$ represent the compositions of $\mathrm{Ga}$ and As, respectively. Apart from the presence of $\mathrm{Ga}$ and As, which are present everywhere, the existence of In and $\mathrm{Sb}$ in the QDs is evident from this result, with an enrichment of In and Sb in the QD cores. This result is definitive as the presence of these four elements is found in all the analyzed QDs. The measured concentration of In and $\mathrm{Sb}$ with the electron probe on the core of the QDs is about double $(\sim 40 \%)$ that in the wetting layer $(\sim 20 \%)$. However, a general problem for the compositional analysis of QDs by TEM and related techniques is that the image corresponds to a projection through the specimen including contributions not only from the QD but also from the material above and below it. The concentration estimates are therefore qualitative in nature, but definitively establish that the $\mathrm{Sb}$ is present within the QDs. The specimen thickness obtained from the low loss EELS analysis is $35-40 \mathrm{~nm}$, somewhat greater than the QD dimensions. Therefore, we estimate that the actual Sb composition of the QD is higher than the measured value that includes the surrounding material, and is likely to be around $60 \%$. We can infer that the cores of the QDs consist of a quaternary $\mathrm{Ga}_{x} \mathrm{In}_{1-x} \mathrm{As}_{y} \mathrm{Sb}_{1-y}$ alloy, with $y \sim 0.4$. This alloy must be the result of an intermixing process during the growth of the GaSb overlayer and the InGaAs alloy that originally comprised the QDs before the GaSb overgrowth was deposited. EDX measurements (not shown here) of these QDs confirm this finding, as the same four elements were

AIP license or copyright; see http://apl.aip.org/apl/copyright.jsp 

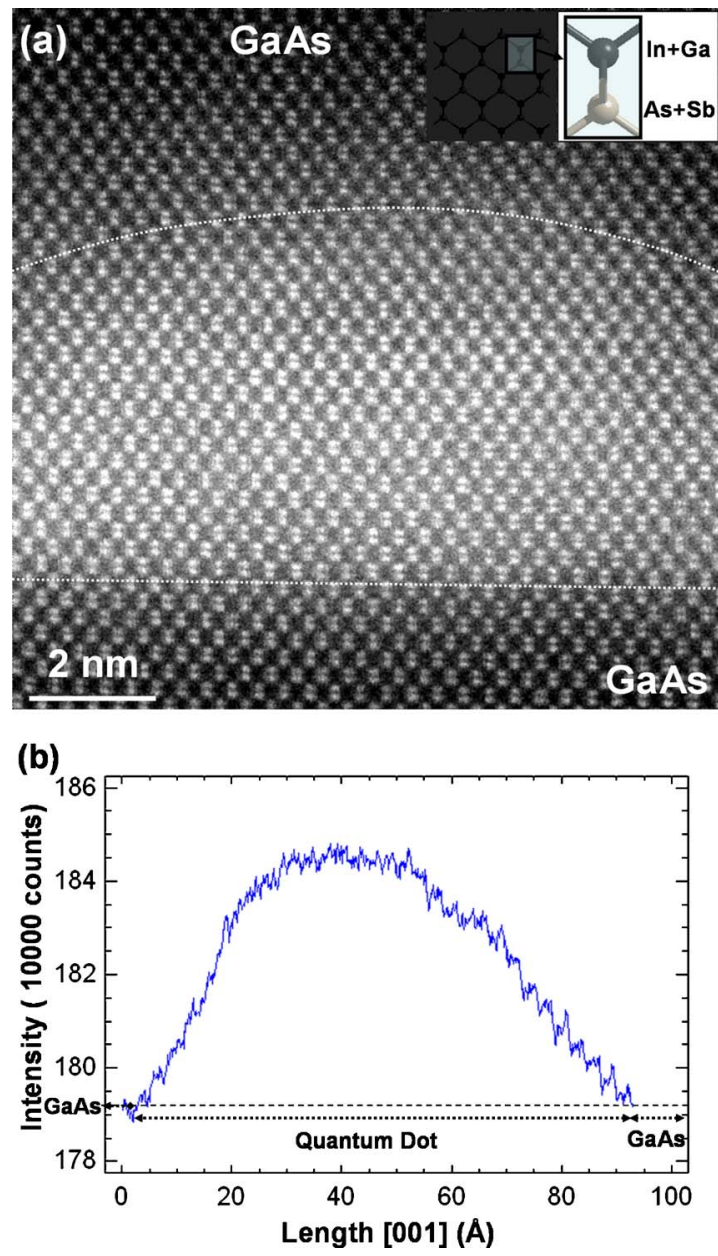

FIG. 3. (a) High resolution HAADF-STEM image of a QD. The approximate location of interfaces with the GaAs substrate and the GaAs capping layer are indicated on the image with dotted lines. The inset at the upper right corner shows the projected atomic structure. (b) Intensity profile taken along [001] across the central part of the HAADF-STEM image of (a).

Figure 3(a) shows a HAADF-STEM image acquired from a QD. The image has been low-pass filtered to eliminate high frequency noise. More intense contrast in this image is associated with high $Z$ numbers, this is, to zones of the material where there are In-rich cationic columns and/or Sbrich anionic columns. Figure 3(b) presents an intensity profile taken across the central part of this image along the [001] growth direction. The shape of this profile with a unique well defined maximum supports our previous findings obtained by EELS. The central region of the QD with a higher intensity is assigned to the $\mathrm{Ga}_{x} \mathrm{In}_{1-x} \mathrm{As}_{y} \mathrm{Sb}_{1-y}$ alloy detected by EELS and no distinct $\mathrm{GaSb}$ capping layer is seen, in agreement with the low magnification HAADF images. The intensities integrated from the central zone of the HAADF image of Fig. 3(a) around the cationic $(\mathrm{In}+\mathrm{Ga})$ and anionic $(\mathrm{As}+\mathrm{Sb})$ column positions have been measured from the raw data image. The maximum intensities for both kinds of atomic columns are located throughout the region where maximum In and $\mathrm{Sb}$ concentrations were detected from low-loss EELS measurements. Furthermore, the depth of field of the aberration-corrected STEM is much reduced compared to the EELS experiments, and despite the strong channelling associated with the high $Z$ elements is estimated to be $10-20 \mathrm{~nm}$.
Therefore the image with brightest contrast is expected to reflect mainly the composition of the QD itself. The ratios between the intensities integrated around the anionic and cationic columns increase from the core outward to the upper part of the QD, in good agreement with the EELS results, which showed $\mathrm{Sb}$ to extend further towards the apex of the QDs with an upper region comprised of $\mathrm{GaAs}_{y} \mathrm{Sb}_{1-y}$ on top of a core of $\mathrm{Ga}_{x} \mathrm{In}_{1-x} \mathrm{As}_{y} \mathrm{Sb}_{1-y}$.

The $x$ and $y$ composition values measured in the QDs from the analysis of the low-loss EEL spectra correspond to compositions inside the miscibility gap of the $\mathrm{Ga}_{x} \mathrm{In}_{1-x} \mathrm{As}_{y} \mathrm{Sb}_{1-y}$ quaternary alloy. ${ }^{15-17}$ In view of this result, we propose that the intermixing process giving rise to the formation of the $\mathrm{Ga}_{x} \mathrm{In}_{1-x} \mathrm{As}_{y} \mathrm{Sb}_{1-y}$ alloy in the core of the QDs is likely to be a strain-driven process.

In summary, InAs and GaSb intermixing has been shown to occur in $\mathrm{GaSb} / \mathrm{InAs}$ QDs grown by MBE on GaAs substrates, resulting in the formation of an $\operatorname{In}_{x} \mathrm{Ga}_{1-x} \mathrm{As}_{y} \mathrm{Sb}_{1-y}$ quaternary alloy in the core of the QDs.

This work was supported by the Office of Basic Energy Sciences, Division of Materials Sciences and Engineering, US DOE (MFC, MV and SJP), the SANDiE European Network of Excellence (Contract No. NMP4-CT-2004-500101), the Spanish MEC (TEC2005-05781-C03-01 y 02, NAN2004 -09109-C04-01, Consolider-Ingenio 2010 CSD2006-00019 and -0004), the CAM (S 0505ESP 0200), and the Junta de Andalucia (PAI research groups TEP-120 and TIC-145; Project No. PAI05-TEP-00383).

${ }^{1}$ V. M. Ustinov and A. E. Zhukov, Semicond. Sci. Technol. 15, R41 (2000).

${ }^{2}$ L. Seravalli, M. Minelli, P. Frigeri, S. Franchi, G. Guizzetti, M. Patrini, T. Ciabattoni, and M. Geddo, J. Appl. Phys. 101, 024313 (2007).

${ }^{3}$ J. M. Ripalda, D. Alonso-Alvarez, B. Alen, A. G. Taboada, J. M. Garcia, Y. Gonzalez, and L. Gonzalez, Appl. Phys. Lett. 91, 012111 (2007).

${ }^{4}$ J. M. Ripalda, D. Granados, Y. Gonzalez, A. M. Sanchez, S. I. Molina, and J. M. Garcia, Appl. Phys. Lett. 87, 202108 (2005).

${ }^{5}$ K. Akahane, N. Yamamoto, S.-I. Gozu, and N. Ohtani, Physica E (Amsterdam) 26, 395 (2005).

${ }^{6}$ H. Y. Liu, M. J. Steer, T. J. Badcock, D. J. Mowbray, M. S. Skolnick, F. Suarez, J. S. Ng, M. Hopkinson, and J. P. R. David, J. Appl. Phys. 99, 046104 (2006).

${ }^{7}$ J. M. Ulloa, I. W. D. Drouzas, P. M. Koenraad, D. J. Mowbray, M. J. Steer, H. Y. Liu, and M. Hopkinson, Appl. Phys. Lett. 90, 213105 (2007). ${ }^{8}$ D. Guimard, S. Tsukamoto, M. Nishioka, and Y. Arakawa, Appl. Phys. Lett. 89, 083116 (2006)

${ }^{9}$ J. C. Harmand, L. H. Li, G. Patriarche, and L. Travers, Appl. Phys. Lett. 84, 3981 (2004).

${ }^{10}$ M. Yano, H. Yokose, Y. Iwai, and M. Inoue, J. Cryst. Growth 111, 609 (1991).

${ }^{11}$ K. Potschke, L. Muller-Kirscha, R. Heitza, R. L. Sellina, U. W. Pohla, D. Bimberg, N. Zakharov, and P. Werner, Physica E (Amsterdam) 21, 606 (2004).

${ }^{12}$ M. Geller, C. Kapteyn, L. Müller-Kirsch, R. Heitz, and D. Bimberg, Appl. Phys. Lett. 82, 2706 (2003).

${ }^{13}$ A. M. Sanchez, M. Gass, A. J. Papworth, and P. J. Goodhew, Phys. Rev. B 70, 035325 (2004).

${ }^{14}$ M. H. Gass, A. J. Papworth, T. J. Bullough, and P. R. Chalker, Ultramicroscopy 101, 257 (2004).

${ }^{15}$ G. B. Stringfellow, J. Cryst. Growth 58, 194 (1982).

${ }^{16}$ J. R. Chang, Y. K. Su, C. L. Lin, K. M. Wu, W. C. Huang, Y. T. Lu, D. H. Jaw, W. L. Li, and S. M. Chen, Appl. Phys. Lett. 75, 238 (1999).

${ }^{17}$ V. A. Elyukhin, R. Peña-Sierra, B. L. Rivera-Flores, and R. Asomoza, J. Phys.: Condens. Matter 16, S2211 (2004). 